
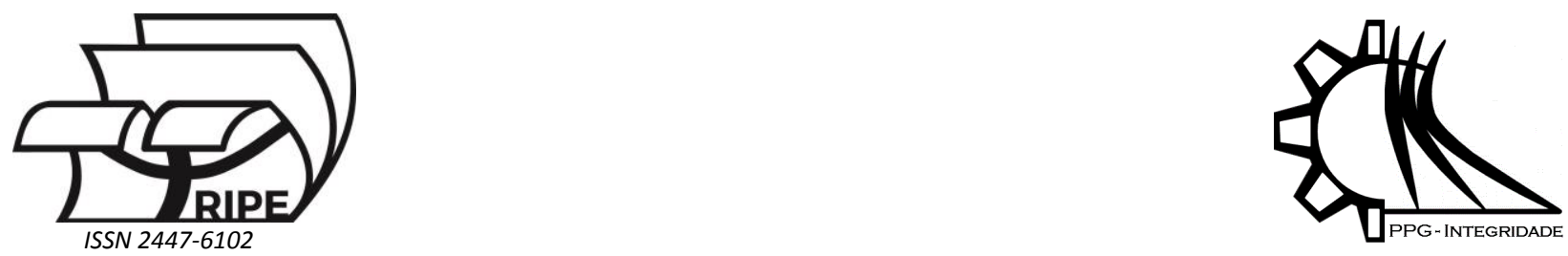

Article

\title{
Estudo numérico para obtenção da razão de aspecto ótima de uma aleta no processo de fusão de material de mudança de fase (PCM)
}

\author{
Becker, F. ${ }^{1}$, Oliveski, R.C. ${ }^{2}$ and Rocha, L.A.O. ${ }^{2, *}$ \\ 1 Fábio Becker - Universidade do Vale do Rio dos Sinos - UNISINOS; fabiobec@edu.unisinos.br \\ 2 Rejane De Césaro Oliveski - Universidade do Vale do Rio dos Sinos - UNISINOS; decesaro@unisinos.br \\ 3 Luiz Alberto Oliveira Rocha - Universidade do Vale do Rio dos Sinos - UNISINOS; luizor@unisinos.br \\ * Correspondente: luizor@unisinos.br
}

Received: 14/12/2018; Accepted: 05/01/2019; Published: 15/01/2019

\begin{abstract}
Resumo: Com a crescente utilização de fontes de energia renováveis, diversos estudos sobre a utilização de materiais de mudança de fase (PCM) ganharam destaque mundial. Muitos destes, concentram-se na sua utilização em cavidades. Para uma cavidade retangular aletada internamente, contendo o PCM ácido láurico em processo de fusão, este trabalho tem por objetivo verificar a influência da razão de aspecto da aleta, bem como, por otimização geométrica, determinar a razão de aspecto ótima, a qual minimizará o tempo do processo de fusão do PCM. Para os casos estudados, a aleta encontra-se posicionada horizontalmente no centro da parede direita da cavidade, sendo suas dimensões alteradas através da variação da sua razão de aspecto, para uma fração de área de 0,01 . As simulações numéricas foram realizadas no software Fluent, com o modelo matemático baseado nas equações da conservação da massa, quantidade de movimento e conservação da energia. No processo de mudança de fase utilizou-se o modelo entalpia-porosidade. Os modelos matemático e numérico foram validados com base em resultados experimentais obtidos da literatura. As malhas computacionais foram avaliadas através do método $\mathrm{GCl}$, onde obteve-se um índice de $1,9 \%$ em relação a malha utilizada. Os resultados obtidos de fração de líquido em função do tempo mostraram que houve uma redução de, aproximadamente, $19 \%$ no tempo total do processo de fusão do PCM, entre as razões de aspecto de 0,026 e 0,6, respectivamente. Dessa forma, para este estudo, obteve-se a menor razão de aspecto como razão de aspecto ótima para a aleta.
\end{abstract}

Palavras-chave: Material de mudança de fase (PCM), Simulação numérica, Otimização, Razão de aspecto.

\section{Numerical study for obtaining the reason of the final appearance of a flip in the fusion process of phase change material (PCM)}

Abstract: With the increasing use of renewable energy sources, several studies on the use of phase change materials (PCM) have gained worldwide prominence. Many of these focus on their use in cavities. For an internally finned rectangular cavity containing the lauric acid PCM in the melting process, this work aims to verify the influence of the fin aspect ratio, as well as, by geometric optimization, determine the optimum aspect ratio, which will minimize the time of the PCM melting process. For the cases studied, the fin is positioned horizontally in the center of the right wall of the cavity, its dimensions being altered by varying its aspect ratio, for a fraction area of 0.01 . Numerical simulations were performed in Fluent software, with the mathematical model based on equations of mass conservation, momentum and energy conservation. In the phase change process the enthalpy-porosity model was used. The mathematical and numerical models were validated based on experimental results obtained from the literature. The computational meshes were evaluated through the $\mathrm{GCl}$ method, where it obtained an index of $1.9 \%$ in relation to the mesh used. The results of liquid fraction as a function of time showed that there was a reduction of 
approximately $19 \%$ in the total time of the PCM fusion process, between the aspect ratios of 0.026 and 0.6 , respectively. Thus, for this study, the lowest aspect ratio was obtained as the optimal aspect ratio for the fin.

Keywords: Phase change material (PCM), Numerical simulation. Optimization, Aspect ratio.

\section{Introdução}

Com o crescente uso de fontes de energia renováveis, estudos com materiais de mudança de fase (PCM - Phase Change Material) apresentam grande importância no cenário mundial. Muitos estudos são direcionados à sua aplicabilidade em cavidades, que podem ser utilizadas em diversos tipos de sistemas. Como exemplo, têm-se sistemas eletrônicos, sistemas de secagem de alimentos, sistemas de condicionadores de ar, entre outros.

A aplicação de PCM em sistemas de condicionadores de ar baseia-se na coleta do ar frio durante o período da noite, para posterior troca de calor com o ar quente do ambiente, durante o dia. Dessa forma, a energia necessária para o resfriamento de ar é reduzida, melhorando a eficiência do sistema de ar condicionado (Zalba et al., 2003).

Pesquisas a respeito da utilização de PCM para melhor aproveitamento de energia térmica, no processo de secagem de batata doce foram alvo de estudo por parte de Devahastin \& Pitaksuriyarat (2006). Esse trabalho consistiu na comparação entre a economia de energia no processo, para diferentes velocidades de entrada de ar no sistema, que tinha como componentes um aquecedor de ar, uma cavidade cilíndrica com PCM e a câmara de secagem, respectivamente. Durante os períodos de maior exposição ao sol, o PCM absorve a energia em excesso, para que quando esta diminua, o processo possa ter continuidade. Os resultados apontaram que foi obtida uma economia de até $40 \%$, com a menor velocidade estudada por eles.

Conforme estudo de Kalbasi \& Salimpour (2015), cavidades contendo PCM podem ser utilizadas para resfriamento de sistemas eletrônicos. Nesse estudo, os autores buscaram determinar o maior tempo de operação do sistema, sem que fosse obtida a temperatura crítica de operação. Há estudos que analisam a aplicabilidade de PCM em cavidades contendo aletas, como o estudo desenvolvido por Kamkari \& Shokouhmand (2014), onde foi analisado o processo de fusão de PCM contido em uma cavidade retangular, com e sem aletas. Nessa análise, os autores observaram que havendo a inclusão de aletas, há uma redução no tempo do processo de fusão do PCM.

Outros estudos sobre o comportamento de PCM em cavidades buscam aliar conceitos da Teoria Construtal, caracterizada pela obtenção de fluxos naturais mais eficientes (Lorente et al., 2015), para otimização das mesmas. Esses estudos se relacionam a essa teoria através de relações de sua geometria, para obtenção de fluxos mais próximos aos eficientes. Como exemplo, as considerações feitas por Kalbasi \& Salimpour (2015), a respeito da análise do comportamento do processo de fusão de PCM através da inclusão de aletas, visando obter o número ótimo de aletas e suas razões de aspecto, no intuito de se obter a temperatura que maximiza o processo de fusão do PCM. Nesse estudo, os autores concluíram que quanto maior o número de aletas, menores podem ser seus comprimentos e que as menores razões de aspecto apresentaram melhor desempenho.

Para uma cavidade retangular aletada internamente, contendo o PCM ácido láurico em processo de fusão, este trabalho tem por objetivo verificar a influência da razão de aspecto da aleta, bem como, por otimização geométrica, por meio do método de pesquisa e implementação da teoria construtal, determinar a razão de aspecto ótima (RAopt), a qual minimizará o tempo do processo de fusão do PCM.

\section{Apresentação do Problema}

A cavidade estudada apresenta geometria retangular aletada, conforme Fig. 1, preenchida com PCM ácido láurico, inicialmente no estado sólido. A mesma possui largura (L) de $50 \mathrm{~mm}$ e altura (H) de $120 \mathrm{~mm}$. A aleta possui largura $L_{1}$ e altura $H_{1}$, e está posicionada horizontalmente no centro da parede direita da cavidade. Na parede superior há uma abertura de $2 \mathrm{~mm}$, rente à parede direita, para simulação da saída do PCM durante o processo de fusão. 


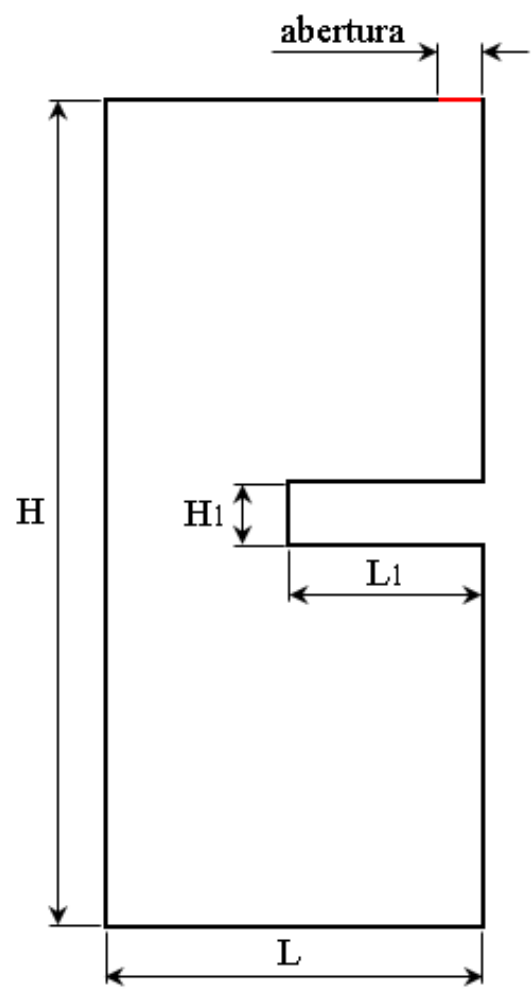

Figura 1. Desenho esquemático do problema.

Para o PCM ácido láurico, os valores do calor específico $\left(c_{p}\right)$, calor latente de fusão $(L)$, massa específica $(\rho)$ e temperaturas do sólido $\left(T_{s}\right)$ e líquido $\left(T_{l}\right)$, respectivamente, estão apresentados na Tab. 1. Os valores da condutividade térmica $(k)$ e da viscosidade dinâmica $(\mu)$ estão apresentados nas Tab. 2 e Tab. 3, respectivamente.

Tabela 1. Propriedades termofísicas do ácido láurico.

\begin{tabular}{lllllll}
\hline$c_{p}[\mathrm{~J} /(\mathrm{kg} \mathrm{K})]$ & & $L[\mathrm{~J} / \mathrm{kg}]$ & $\rho\left[\mathrm{kg} / \mathrm{m}^{3}\right]$ & & $T[\mathrm{~K}]$ & \\
\hline$s$ & $I$ & & $s$ & $l$ & $s$ & 321,35 \\
\hline 2180 & 2390 & 187210 & 940 & 885 & 316,65 & \\
\hline
\end{tabular}

Fonte: Kamkari \& Shokouhmand (2014).

Tabela 2. Condutividade térmica do ácido láurico.

\begin{tabular}{l|llllllll}
\hline$T[\mathrm{~K}]$ & 293 & 303 & 313 & 323 & 328 & 333 & 338 & 343 \\
\hline$k[\mathrm{~W} /(\mathrm{m} \mathrm{K})]$ & 0,161 & 0,159 & 0,158 & 0,145 & 0,143 & 0,142 & 0,139 & 0,138 \\
\hline
\end{tabular}

Fonte: Shokouhmand \& Kamkari (2013).

Tabela 3. Viscosidade dinâmica do ácido láurico.

\begin{tabular}{l|lllllll}
\hline$T[\mathrm{~K}]$ & 321,2 & 322 & 333 & 344 & 355 & 372 & 383 \\
\hline$\mu[\mathrm{kg} /(\mathrm{m} \mathrm{s})]$ & 0,00760 & 0,00747 & 0,00542 & 0,00428 & 0,00338 & 0,00250 & 0,00210 \\
\hline
\end{tabular}

Fonte: Valeri \& Meirelles (1997).

\section{Abordagem Teórica}

A abordagem teórica é desenvolvida através de duas metodologias: simulação numérica por CFD e otimização geométrica através da aplicação do método de pesquisa, com a utilização da Teoria Construtal, as quais são apresentas na sequência. 


\subsection{Simulação Numérica}

O modelo matemático consiste na equação da conservação da massa, conforme Eq. (1), quantidade de movimento, conforme Eq. (2) e conservação de energia, conforme Eq. (3), acrescidas do modelo de mudança de fase.

$$
\begin{gathered}
\frac{\partial \rho}{\partial t}+\nabla(\rho \vec{V})=0 \\
\frac{\partial \rho \vec{V}}{\partial t}+\nabla(\rho \vec{V} \vec{V})=-\nabla p+\nabla(\mu \nabla \vec{V})+\rho \vec{g}+S \\
\frac{\partial(\rho \lambda)}{\partial t}+\nabla(\rho \vec{V} \lambda)=\nabla(k \nabla T)
\end{gathered}
$$

onde $\vec{V}$ é o vetor velocidade, $t$, o tempo, $p$, a pressão, $\vec{g}$, a aceleração da gravidade, $S$, o termo fonte e $\lambda$, a entalpia total, obtida pela soma da variação da entalpia na mudança de fase $\left(\lambda_{L}\right)$ e pela entalpia sensível $\left(\lambda_{s e}\right)$, obtida através da Eq. (4) (Shmueli et al., 2010).

$$
\lambda_{s e}=\lambda_{\text {ref }}+\int_{T_{r e f}}^{T} C_{p} d T
$$

onde $\lambda_{\text {ref }}$ é a entalpia na temperatura de referência $\left(T_{\text {ref }}\right)$. Já a entalpia na mudança de fase é calculada pela Eq. (5).

$$
\lambda_{L}=\beta L
$$

O modelo de mudança de fase é o da entalpia-porosidade, o qual trata a zona de interface sólido-líquido (mushy zone) como uma zona porosa, sendo o termo $S$ descrito conforme a Eq. (6) (Assis et al., 2007).

$$
S=\frac{(1-\beta)^{2}}{\left(\beta^{3}+\varepsilon\right)} C \vec{V}
$$

onde 6 é a fração líquida, $\varepsilon$ é uma constante de valor 0,001 , utilizada para evitar divisão por zero e $C$ é a constante da zona porosa, dependente da morfologia da zona porosa (Voller \& Prakash, 1987). A fração líquida durante o processo de mudança de fase é obtida através da Eq. (7).

$$
\beta=\left\{\begin{array}{rll}
0 & \text { se } & T<T_{s} \\
1 & \text { se } & T>T_{l} \\
\frac{T-T_{s}}{T_{l}-T_{s}} & \text { se } & T_{s}<T<T_{l}
\end{array}\right.
$$

Os softwares utilizados para este estudo foram: ANSYS ICEM ${ }^{\circ}$, para criação das malhas computacionais, ANSYS FLUENT ${ }^{\circledR}$, para realização das simulações numéricas e ANSYS CFD-Post , para realização do pós-processamento. Na correção da pressão foi utilizado o esquema PRESTO e no acoplamento da pressão-velocidade o esquema SIMPLE. Nas equações de energia e quantidade de movimento foi utilizado First Order Upwind, com critério de convergência $10^{-8}$ para energia e $10^{-5}$ para velocidade e continuidade.

As paredes inferior, superior e esquerda da cavidade foram consideradas adiabáticas. Já a condição de contorno da aleta e da parede direita foi de temperatura prescrita e uniforme: $T_{a}=343 \mathrm{~K}$. A condição inicial do PCM foi: $T_{i}=298$ K. Para a abertura de $2 \mathrm{~mm}$ foi utilizada condição de Pressure Outlet, com temperatura de $343 \mathrm{~K}$.

\subsection{Otimização}

A otimização geométrica da aleta será determinada através do método de pesquisa com a aplicação da Lei Construtal. Pela utilização desta, as melhores configurações poderão ser obtidas com a determinação dos melhores fluxos (Lorente et al., 2015). Dessa forma, para este estudo, a geometria da aleta que caracteriza o melhor fluxo é a que apresenta o menor tempo do processo de fusão, o que resultará na razão de aspecto ótima (RA opt $_{\text {) }}$. Para obtenção desta, diferentes razões de aspecto de aleta $\left(R A_{1}\right)$ são analisadas. A razão de aspecto da aleta $\left(R A_{1}\right)$ é a razão entre a $H_{1}$ e $L_{1}\left(R A_{1}=H_{1} / L_{1}\right)$, para uma fração de área $(\phi)$ de 0,01 . A fração de área é a razão entre a área da aleta e a área da cavidade $\left(\phi=A_{a} / A\right)$, sendo as áreas constantes e iguais a $60 \mathrm{~mm}^{2}$ e $6000 \mathrm{~mm}^{2}$, respectivamente. As dimensões da aleta, com suas respectivas $\mathrm{RA}_{1}$, estão expostas na Tab. 4. 
Tabela 4. Dimensões da aleta.

\begin{tabular}{llllllllll}
\hline $\mathrm{L}_{1}[\mathrm{~mm}]$ & 10 & 15 & 20 & 25 & 30 & 35 & 40 & 45 & 48 \\
\hline $\mathrm{H}_{1}[\mathrm{~mm}]$ & 6,00 & 4,00 & 3,00 & 2,40 & 2,00 & 1,71 & 1,50 & 1,33 & 1,25 \\
\hline $\mathrm{RA}_{1}[-]$ & 0,600 & 0,267 & 0,150 & 0,096 & 0,066 & 0,049 & 0,037 & 0,029 & 0,026 \\
\hline
\end{tabular}

\section{Resultados e Discussões}

Os resultados aqui apresentados dizem respeito à validação numérica e ao estudo de caso proposto.

\subsection{Validação Numérica}

A validação numérica foi realizada com base no estudo experimental de Kamkari \& Shokouhmand (2014). Inicialmente foram criadas três malhas computacionais, contendo: 28457, 14520 e 10118 elementos, representadas na Fig. 2. A análise da independência de malha foi realizada pelo método GCl (Celik et al., 2008). Através desse método, foi realizado o comparativo entre a malha intermediária com a mais refinada e menos refinada, o que resultou em 1,9\% e 5,5\%, respectivamente. Dessa forma, a malha selecionada foi a intermediária, que contém 14520 elementos.

(a)

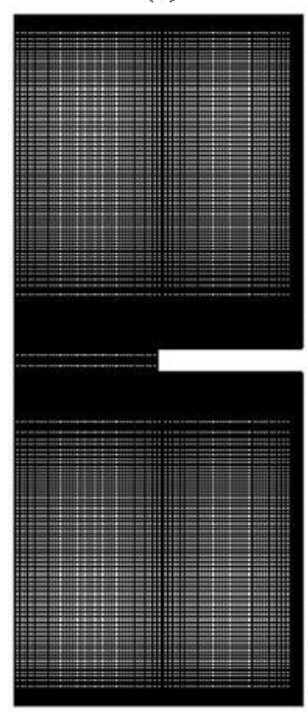

(b)

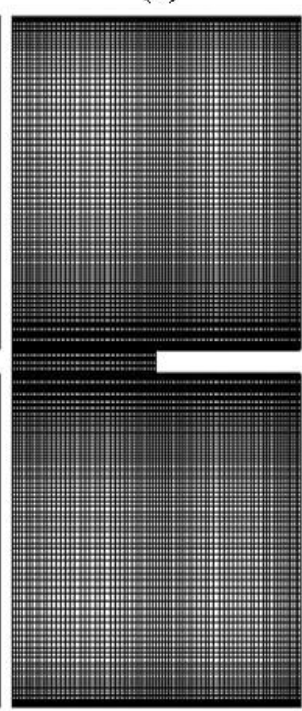

(c)

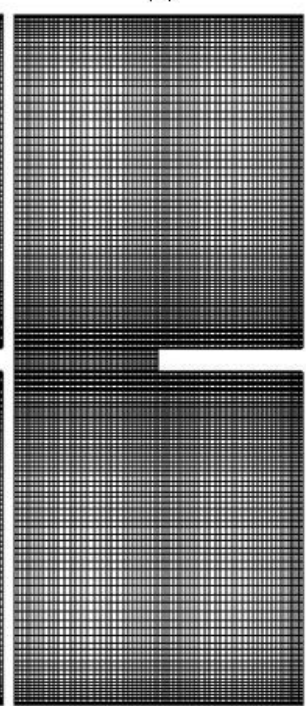

Figura 2. Malhas computacionais: (a) 28457, (b) 14520 e (c) 10118 elementos.

As Figs. 3(a-d) apresentam campos de fração de líquido (b) para os tempos de 10 e 30 min, respectivamente, obtidos por Kamkari \& Shokouhmand (2014), Fig. 3(a, c), e o presente estudo, Fig. 3(b, d). As cores branca e azul representam o PCM no estado sólido e as cores preta e vermelha representam o PCM no estado líquido. Comparando-se os resultados experimentais e numéricos, tanto para o $t=10$ min quanto para $0 t=30$ min, observa-se uma boa concordância entre os campos de fração de líquido. 
$\mathrm{t}=10 \mathrm{~min}$

(a)

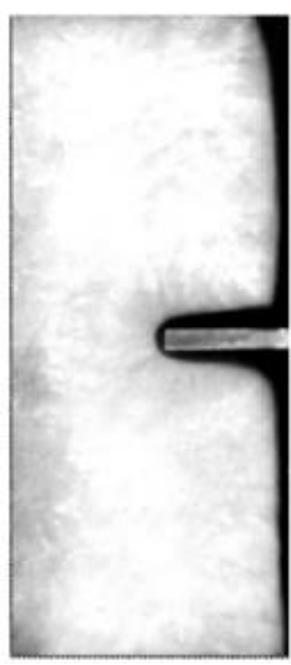

(b)

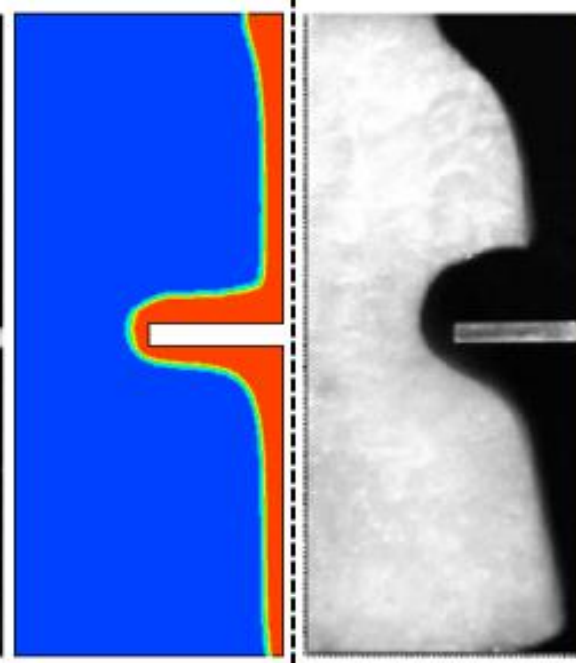

$\mathrm{t}=30 \mathrm{~min}$

(c)

(d)

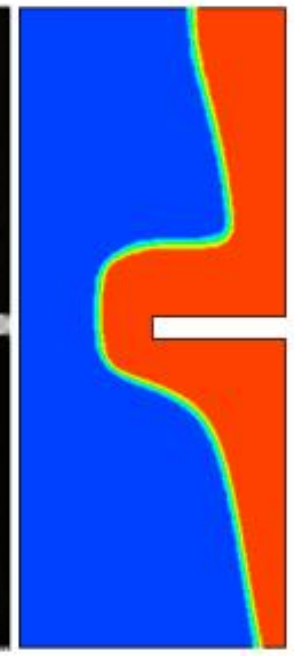

Figura 3. Campos de 6 para $\mathrm{t}=10$ e 30 min para: (a) experimental de Kamkari \& Shokouhmand (2014), (b) este estudo, (c) experimental de Kamkari \& Shokouhmand (2014) e (d) este estudo.

Na Fig. 4 são apresentados perfis de $B$ ao longo do tempo, obtidos pelo presente estudo e resultados experimentais de Kamkari \& Shokouhmand (2014). Ao longo do tempo, o erro médio, para o afastamento entre resultados foi de, aproximadamente, 5,4 \%, enquanto que o máximo afastamento foi de, aproximadamente, 6,3\%, para $B=0,856 \mathrm{et}=110 \mathrm{~min}$.

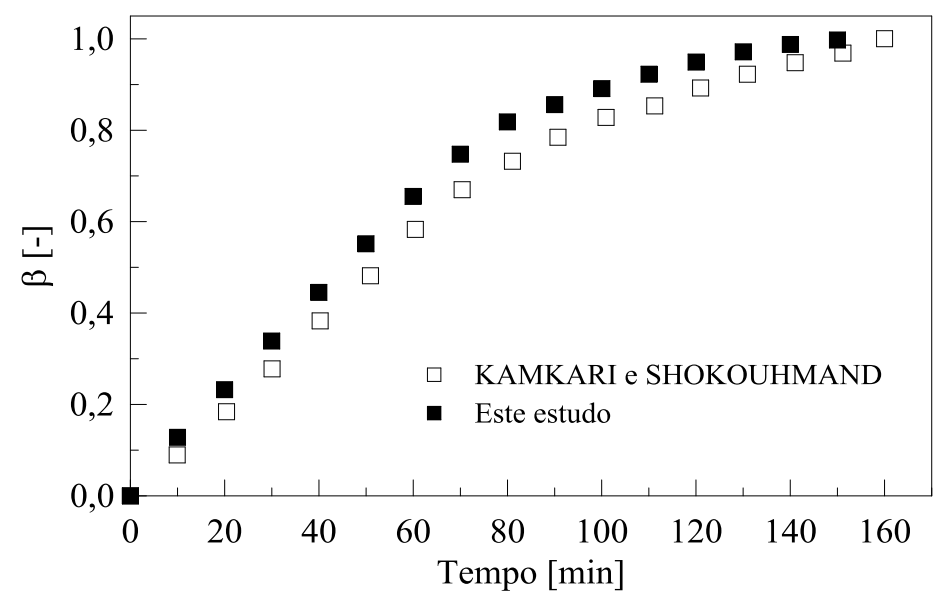

Figura 4. 6 pelo tempo: experimental de Kamkari \& Shokouhmand (2014) e este estudo.

Considerando-se a boa concordância entre os resultados experimentais de Kamkari \& Shokouhmand (2014) e os numéricos do presente trabalho, tanto na forma qualitativa (Fig. 3) quanto quantitativa (Fig. 4), considera-se então o modelo validado e apto a ser utilizado para o Estudo de Caso.

\subsection{Estudo de Caso}

As Figs. 5(a-c) apresentam campos de 6 para tempos de 10, 30, 50, 70, 90 e 110 min, para três tamanhos de aletas. Pode-se observar que, para qualquer tempo, a fusão ocorreu mais rapidamente para o caso com $R A_{1}=0,026,0$ qual apresenta maior área de transferência de calor. 


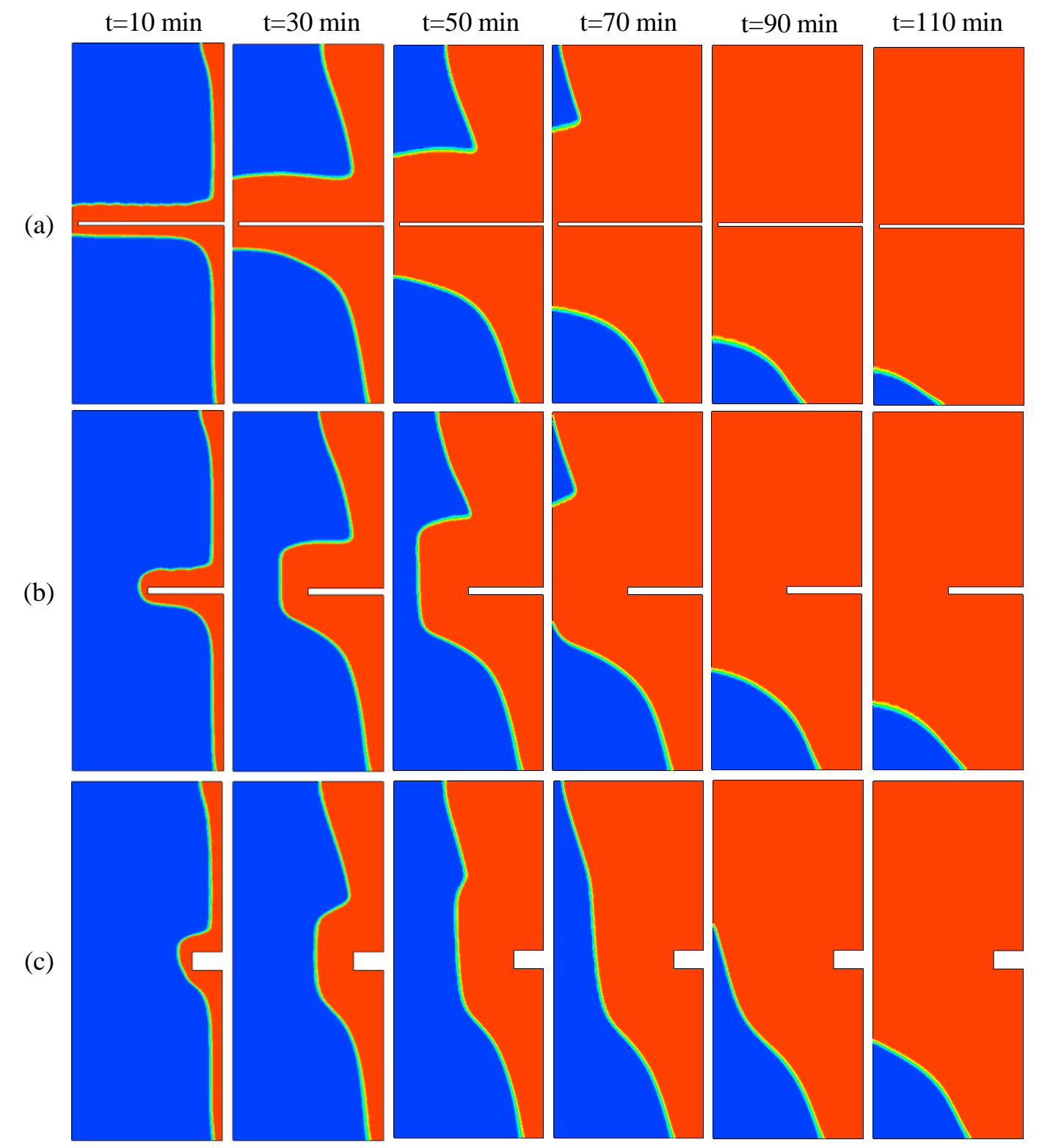

Figura 5. Campos de 6 para $t=10,30,50,70,90$ e 110 min para: (a) $R A_{1}=0,026$; (b) $R A_{1}=0,096$; (c) $R A_{1}=0,600$.

Na Fig. 6 estão apresentados valores de perfis de $B$ em função do tempo para as diferentes $\mathrm{RA}_{1}$. Nela observa-se uma tendência de redução do tempo total do processo de fusão, com a redução dos valores de $\mathrm{RA}_{1}$. Isso ocorre devido ao aumento do perímetro da aleta, aumentando, dessa forma, a área de transferência de calor entre a aleta e o PCM. Por exemplo, o tempo para o PCM atingir $B=0,8$ foi de, aproximadamente, 66 e de 90 min, para $\mathrm{RA}_{1}=0,026$ e 0,6, respectivamente. 


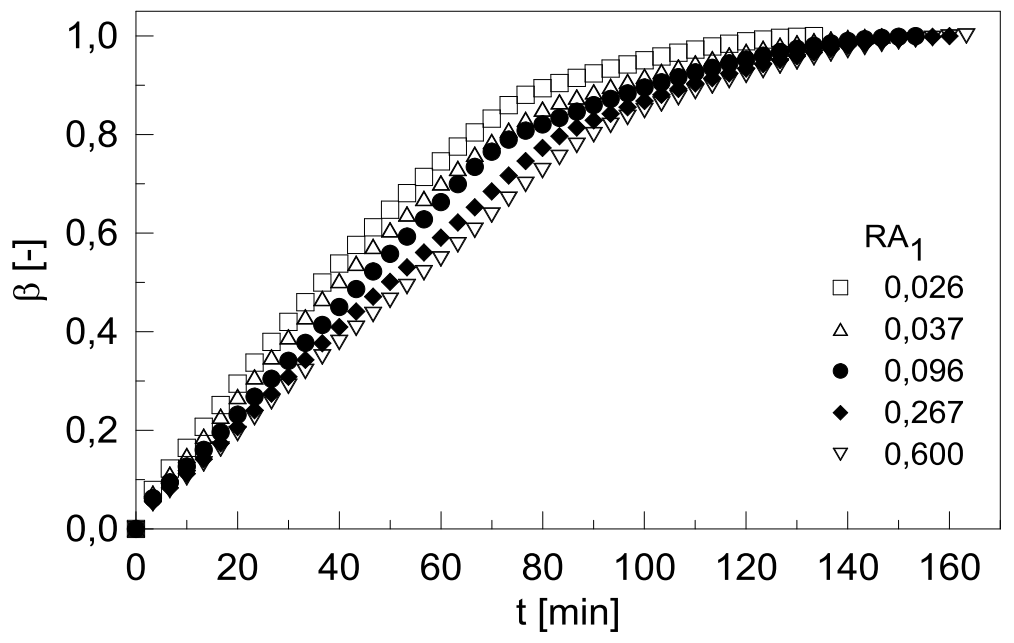

Figura 6. 6 x tempo para diferentes $\mathrm{RA}_{1}: 0,026 ; 0,037 ; 0,096 ; 0,267 ; 0,600$.

A Fig. 7 mostra, para várias $\mathrm{RA}_{1}$, o tempo necessário para que o PCM passe da fase sólida para a fase líquida, ou seja, para que atinja a condição de $B=1$. Nessa figura observa-se que, quanto menor é o valor da $R A_{1}, m^{2}$ enor é o tempo necessário para o PCM atingir a condição de fusão completa. Enquanto a aleta com $\mathrm{RA}_{1}=0,026$ leva, aproximadamente, 135 min para fundir completamente, a aleta com RA $\mathrm{R}_{1}=0,6$ leva, aproximadamente, 166 min, o que corresponde a uma diferença de, aproximadamente, $19 \%$. Dessa forma, através dos resultados apresentados, se obteve como $\mathrm{RA}_{\mathrm{opt}}$ da aleta, a $\mathrm{RA}_{1}=0,026$ para $\phi$ de 0,01 .

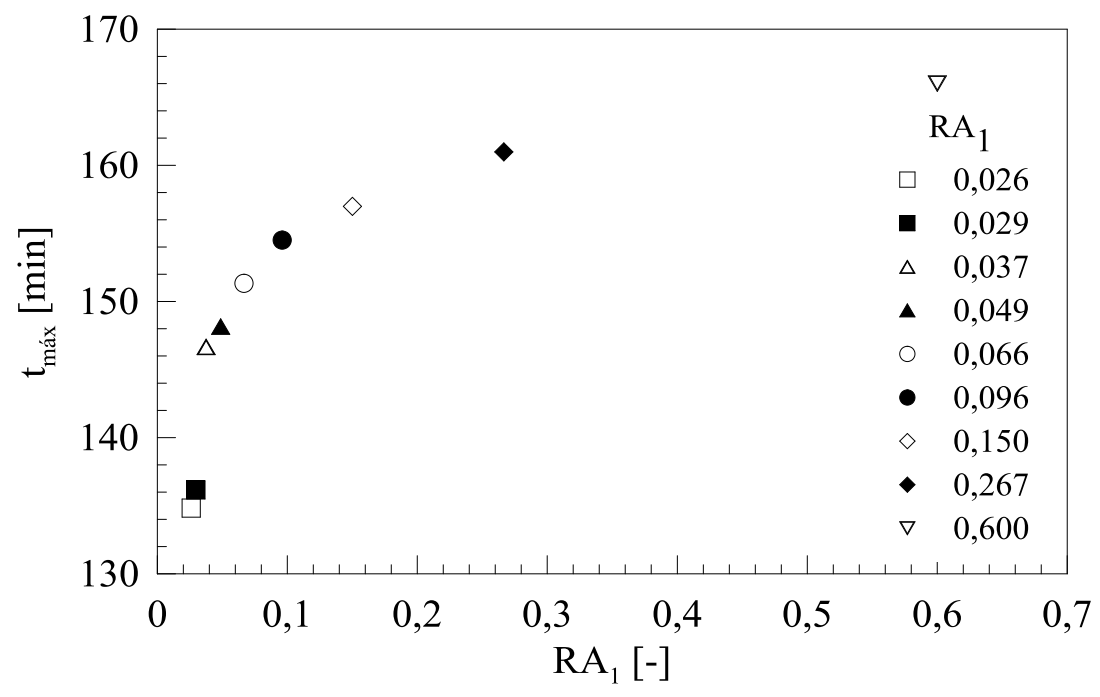

Figura 7. Tempo de fusão total para as diferentes $R A_{1}$.

\section{Conclusões}

Esse trabalho apresentou um estudo numérico para análise do processo de fusão de PCM, contido em uma cavidade retangular aletada. O objetivo desse estudo foi determinar a $\mathrm{RA}_{\mathrm{opt}}$ da aleta, por meio de otimização geométrica e implementação da teoria construtal, para razões de aspecto de 0,026 até 0,6 , com a qual fosse possível obter o menor tempo do processo de fusão do PCM, para a respectiva cavidade.

Verificou-se que os resultados qualitativos e quantitativos estão de acordo com os resultados experimentais apresentados por Kamkari \& Shokouhmand (2014), validando, dessa forma, o modelo e metodologia utilizados.

Em relação ao estudo de caso, os resultados preliminares de campos e perfis de fração de líquido em função do tempo revelaram que o processo de fusão de PCM apresentou menor tempo para a menor $\mathrm{RA}_{1}$ analisada. Nesse caso, obteve-se uma redução de, aproximadamente, $19 \%$ no tempo total do processo, da $\mathrm{RA}_{1}=0,6$ à 0,026 , respectivamente. Dessa forma, obteve-se como $\mathrm{RA}_{\text {opt }}$ da aleta a $\mathrm{RA}_{1}=0,026$. Este resultado concorda com resultados 
encontrados na literatura para problemas com cavidade sólida, onde, para cavidades de baixo valor da fração de área, a razão de aspecto ótima é aquela que penetra quase completamente o sólido conforme Biserni et al. (2003).

Agradecimentos: Os autores agradecem à UNISINOS (Universidade do Vale do Rio dos Sinos), à CAPES (Coordenação de Aperfeiçoamento de Pessoal de Nível Superior) e à FAPERGS (Fundação de Amparo à Pesquisa do Estado do Rio Grande do Sul) pelo apoio prestado à realização dessa pesquisa.

O presente trabalho foi realizado com apoio da Coordenação de Aperfeiçoamento de Pessoal de Nível Superior - Brasil (CAPES) Código de Financiamento 001.

\section{Referências}

1. Assis, E., Katsman, L., Ziskind, G., \& Letan, R. (2007). Numerical and experimental study of melting in a spherical shell. International Journal of Heat and Mass Transfer, 50, 1790-1804. doi:10.1016/j.ijheatmasstransfer.2006.10.007

2. Biserni, C., Rocha, L. A. O., \& Bejan, A. (2003). Inverted fins: geometric optimization of the intrusion into a conducting wall. International Journal of Heat and Mass Transfer, 12-13, pp. 2577-2586. doi:10.1016/j.ijheatmasstransfer.2003.12.018

3. Celik, I. B., Ghia, U., Roache, P. J., Freitas, C. J., Coleman, H., \& Raad, P. E. (2008). Procedure for Estimation and Reporting of Uncertainty Due to Discretization in CFD Applications. Journal of Fluids Engineering, 130. doi:10.1115/1.2960953

4. Devahastin, S., \& Pitaksuriyarat, S. (2006). Use of latent heat storage to conserve energy during drying and its effect on drying kinetics of a food product. Applied Thermal Engineering, 26, pp. $1705-1713$. doi:10.1016/j.applthermaleng.2005.11.007

5. Kalbasi, R., \& Salimpour, M. R. (2015). Constructal design of horizontal fins to improve the performance of phase change material rectangular enclosures. Applied Thermal Engineering, 91, pp. $234-244$. doi:10.1016/j.applthermaleng.2015.08.024

6. Kalbasi, R., \& Salimpour, M. R. (2015). Constructal design of phase change material enclosures used for cooling electronic devices. Applied Thermal Engineering, 84, pp. 339-349. doi:10.1016/j.applthermaleng.2015.03.031

7. Kamkari, B., \& Shokouhmand, H. (2014). Experimental investigation of phase change material melting in rectangular enclosures with horizontal partial fins. International Journal of Heat and Mass Transfer, 78, pp. 839-851. doi:10.1016/j.ijheatmasstransfer.2014.07.056

8. Lorente, S., Bejan, A., \& Niu, J. L. (2015). Constructal design of latent thermal energy storage with vertical spiral heaters. International Journal of Heat and Mass Transfer, 81, pp. $283-288$. doi:10.1016/j.ijheatmasstransfer.2014.09.077

9. Shmueli, H., Ziskind, G., \& Letan, R. (2010). Melting in a vertical cylindrical tube: Numerical investigation and comparison with experiments. International Journal of Heat and Mass Transfer, 53, pp. $4082-4091$. doi:10.1016/j.ijheatmasstransfer.2010.05.028

10. Shokouhmand, H., \& Kamkari, B. (2013). Experimental investigation on melting heat transfer characteristics of lauric acid in a rectangular thermal storage unit. Experimental Thermal and Fluid Science, 50, $201-212$. doi:10.1016/j.expthermflusci.2013.06.010

11. Valeri, D., \& Meirelles, A. J. A. (1997). Viscosities of Fatty Acids, Triglycerides, and Their Binary Mixtures. Journal of the American Oil Chemists' Society, 74, 1221-1226.

12. Voller, V. R., \& Prakash, C. (1987). A fixed grid numerical modelling methodology for convection-diffusion mushy region phase-change problems. International Journal of Heat and Mass Transfer, 30, pp. 1709-1719.

13. Zalba, B., Marín, J. M., Cabeza, L. F., \& Mehling, H. (2003). Review on thermal energy storage with phase change: materials, heat transfer analysis and applications. Applied Thermal Engineering, 23, pp. 251-283. 